\title{
Kafkas Erzählung In der Strafkolonie und ihre »unverwischbaren Fehler«
}

\author{
Bernd W. Seiler
}

Online publiziert: 14. Februar 2020

(C) Der/die Autor(en) 2020

Zusammenfassung Noch nie ist genauer bedacht worden, auf welche »Fehler « Kafka in seiner Strafkolonie-Erzählung aufmerksam geworden ist. Geht man der Frage nach, erkennt man, dass ein »unverwischbarer« Widerspruch die gesamte Handlung durchzieht: Einerseits ist das Hinrichtungsverfahren in der Strafkolonie allgemein bekannt, andererseits dürfen die Verurteilten nichts darüber wissen, wenn es seinen Zweck erfüllen soll. Woher kommen dann die vielen ahnungslosen Delinquenten, die der Offizier mit seinem »Apparat« hinrichtet? Diese im Text nie berührte Unklarheit hat weitere Widersprüche zur Folge und lässt überdies erkennen, dass man sich auch auf den Reisenden als Beurteiler nicht verlassen kann. Eine befriedigende Auflösung der Unstimmigkeiten findet sich nicht. Es gibt da tatsächlich einen »Wurm, der selbst das Volle der Geschichte hohl macht«, wie Kafka selbst festgestellt hat.

Der Aufsatz bündelt eine Analyse, die bereits einem Materialienband für den Literaturunterricht zugrunde gelegt worden ist: Bernd W. Seiler, Franz Kafka: In der Strafkolonie. Ein Hungerkünstler, Braunschweig 2019.

B. W. Seiler $(\bowtie)$

Schöneberger Str. 23, 33619 Bielefeld, Deutschland

E-Mail: bseiler@uni-bielefeld.de 


\title{
Kaka's narrative The Penal Colony and its »indelible mistakes«.
}

\begin{abstract}
Serious consideration has never been given to the »mistakes « which aroused Kafka's attention in his narrative, The Penal Colony. An exact investigation indicates that an »indelible« contradiction permeates the entire plot: On the one hand, the special kind of execution is generally known throughout the penal colony; on the other hand, the condemned are not supposed to know of it so that it can serve its purpose. So where do the many unknowledgeable delinquents come from, to be executed by the officer with his »apparatus«? This ambiguity, which is never touched upon in the text, results in further contradictions and also shows that one cannot rely upon the traveler as an evaluator. There is no satisfactory solution of the inconsistencies: it is really a »worm « in the story »which hollows out the substance of it«, as Kafka himself determined.
\end{abstract}

Die vielen Zweifel, die Franz Kafka seinen Werken, ja schon seinem Schreiben gegenüber hatte, sind bekannt. Kaum je war er mit dem, was er zu Papier gebracht hatte, zufrieden. Auch die Zustimmung von Lesern oder Zuhörern konnte ihn nicht beeindrucken, sobald ihm selbst etwas unzureichend erschien. Einen »besseren Kritiker als mich während des Vorlesens « gebe es nicht, bemerkt er, als er seinen Eltern den Heizer darbietet, und registriert darin »viele flache Stellen vor offenbar unzugänglichen Tiefen $\ll .{ }^{1}$ Ebenso kann ihn ein Lob, das er zu dem Band Betrachtung von einem Schriftsteller-Kollegen erhält, von seiner eigenen Skepsis nicht abbringen. Für einen Augenblick habe er zwar geglaubt, sein Buch sei »wirklich gut«, erklärt er Felice Bauer, habe dann aber erkennen müssen, dass sich die zustimmende Beurteilung nur »vollständigem Mißverständnis« verdankte. ${ }^{2}$

Besonders bezweifelt hat Kafka die Qualität der Erzählung In der Strafkolonie. Niedergeschrieben im Oktober 1914, als er sich erstmals allein in der Wohnung seiner Schwester Elly einrichten konnte, findet er sie anfangs wohl noch geglückt. »14 Tage, gute Arbeit zum Teil« notiert er für die Zeit ihrer Entstehung. ${ }^{3}$ Als er den Text einige Wochen später bei Franz Werfel vorliest - anwesend sind auch die Freunde Max Brod und Otto Pick sowie Werfels »schöne Schwestern« -, ist er mit dem Resultat aber nur noch »nicht ganz unzufrieden « und ergänzt: »bis auf die überdeutlichen unverwischbaren Fehler «. ${ }^{4}$ Gleichwohl bietet er das Werk dem Verleger Kurt Wolff zum Druck an und liest es am 10. November 1916 in München vor. ${ }^{5}$ Als sich jedoch die Drucklegung ein Jahr später konkretisiert, zögert

\footnotetext{
1 Franz Kafka, Tagebücher, hrsg. Hans-Gerd Koch, Michael Müller, Malcolm Pasley, Frankfurt a.M. 2002, 561 (24. Mai 1913).

2 Franz Kafka, Briefe an Felice, hrsg. Erich Heller, Jürgen Born, Frankfurt a.M. 1967, 278 (31. Januar 1913).

3 Kafka (Anm. 1), 678 (15. Oktober 1914).

4 Kafka (Anm. 1), 703 (2. Dezember 1914).

5 Kafka (Anm. 2), 744 (Postkarte vom 7. Dezember 1916: » ... habe nach 2jährigem Nichtschreiben den phantastischen Übermut gehabt, öffentlich vorzulesen, während ich seit 11/2 Jahren in Prag meine[n] besten Freunden nichts vorgelesen habe.«).
} 
er. »Niemals habe ich aus ganz freiem Herzen die Veröffentlichung dieser Geschichte verlangt«, schreibt er an Kurt Wolff. »Zwei oder drei Seiten kurz vor ihrem Ende sind Machwerk, ihr Vorhandensein deutet auf einen tieferen Mangel, es ist da irgendwo ein Wurm, der selbst das Volle der Geschichte hohl macht.« Er werde »bessere Arbeiten« abliefern können, wenn er bei Kräften bleibe. ${ }^{6}$ Im Oktober 1919 kommt es dann aber doch zur Veröffentlichung, nachdem Kafka - vermutlich gegen den Schluss hin - »ein kleines Stück herausgenommen « hat. $^{7}$

Wie ist die Kafka-Literatur mit diesen Selbstzweifeln umgegangen? Im Großen und Ganzen, so muss man feststellen, hat sie sie ignoriert oder sich mit ihren Gründen nicht näher befasst. »Es lässt sich natürlich nicht herausfinden, was ihn an seiner Erzählung gestört hat «, heißt es beispielsweise bei Kremer. ${ }^{8}$ Allenfalls wird das für Kafka Unbefriedigende in der Schlussgestaltung vermutet. Ähnlich wie für die Verwandlung habe er wohl den »Perspektivenwechsel « gemeint, in dem der Abgang des Reisenden von der Insel erzählt werde. ${ }^{9}$ Oder man sieht das Problem in der Passivität des Reisenden, weil dieser entgegen seinen Grundsätzen nichts weiter tut, als die Flucht zu ergreifen. ${ }^{10}$ Indessen sind das eigentlich keine Fehler, sondern durchaus nachvollziehbare Erzählmomente, die man lediglich vom Gesamteindruck her vielleicht für nicht geglückt halten könnte. ${ }^{11}$

Um aber zunächst den Inhalt zu rekapitulieren: Ein Reisender, der sich von den Rechtszuständen in verschiedenen Weltgegenden ein Bild machen will, kommt auf eine Tropeninsel, auf der sich eine Strafkolonie befindet. Er soll der Hinrichtung eines Soldaten beiwohnen, der einen Vorgesetzten angegriffen hat. Der ausführende Offizier erklärt ihm, dass die Tötung mittels einer Stechmaschine erfolgt, die dem Verurteilten das übertretene Gebot in einer zwölf Stunden dauernden Prozedur auf den Rücken schreibt, bis er verblutet ist. Der Wert dieser Methode: Der Verurteilte entziffert nach und nach, woran er sich hätte halten müssen, und erlebt diese Einsicht als ein höchstes Glück. Die Hinrichtungen haben deshalb über lange Zeit hin auch ein großes Publikum gehabt, immer wieder wollten die Menschen dabei sein, wenn ein Verurteilter seine Schuld erfasste.

Seit dem Tod des alten Kommandanten ist das Interesse an den Hinrichtungen aber zurückgegangen, und der neue Kommandant scheint zu beabsichtigen, sie in

\footnotetext{
${ }^{6}$ Franz Kafka, Briefe 1902-1924, hrsg. Max Brod, Frankfurt a.M. 1958, 159. (4. September 1917 an Kurt Wolff).

7 Kafka (Anm. 6), 245 (11. November 1918 an Kurt Wolff). Da sich das Manuskript der Strafkolonie nicht erhalten hat, lässt sich über die ursprüngliche Fassung nichts sagen.

8 Detlef Kremer, Kafka. Die Erotik des Schreibens, Frankfurt a.M. 1989, 150.

9 Peter-André Alt, Franz Kafka. Der ewige Sohn, München ${ }^{2} 2008$, 475. Ein »Perspektivenwechsel« liegt allerdings gar nicht vor, sondern es öffnet sich lediglich der Horizont zu der Strafkolonie hin, die an dem Hinrichtungsplatz ohnedies gegenwärtig ist. Zutreffend beurteilt bei Alexander Honold, »In der Strafkolonie«, in: Bettina von Jagow, Oliver Jahraus (Hrsg.), Kafka-Handbuch, Göttingen 2008, 477-503, hier: 481.

10 Ausgeführt bei Heinz Politzer, Franz Kafka, der Künstler, Frankfurt a.M. 1965, $174 \mathrm{ff.}$

11 Die Diskussion resümierend schreibt Susanne Kaul, Einführung in das Werk Franz, Kafkas, Darmstadt 2010, 116, Kafka störe anscheinend das »Sprunghafte« des Schlusses, »da es die Einheit des Spannungsbogens aufbricht. «In der Tat hat Kafka 1917 in seinem Tagebuch mehrere andere Szenen entworfen, die den Schluss hätten ersetzen oder ergänzen können, nur dass sich keine von ihnen an den vorhandenen Text anschließen lässt. Kafka (Anm. 1), 822-827 (1917).
} 
dieser Form überhaupt zu verbieten. Der ausführende Offizier will den Reisenden deshalb dafür gewinnen, sich zum Fürsprecher des Verfahrens zu machen. Als er aber erkennen muss, dass ihm das nicht gelingt, trifft er eine überraschende Entscheidung. Er gibt den Verurteilten, dessen Martyrium gerade beginnt, frei und bereitet seine Selbsthinrichtung vor. »Sei gerecht!« lautet der Schuldspruch, mit dem er sich töten lassen will. Doch die Maschine arbeitet nicht, wie der Reisende es erwartet. Sie tötet den Offizier schon in wenigen Minuten und zerlegt sich währenddessen selbst. Der Reisende, beeindruckt von dem Selbstopfer des Offiziers, lässt sich in der Strafkolonie noch das Grab des alten Kommandanten zeigen und eilt dann zu dem Boot, mit dem er die Insel verlassen will. Den Verurteilten und seinen Bewacher, die sich ihm anzuschließen versuchen, weist er zurück.

Sieht man zunächst ab von dem, was Kafka selbst mit den »überdeutlichen Fehlern « gemeint haben könnte, so ist eine rätselhafte Unklarheit zunächst darin auszumachen, dass die Beteiligten unterschiedliche Sprachen sprechen. Der Reisende unterhält sich mit dem Offizier auf Französisch, erkennt aber, dass »weder der Soldat noch der Verurteilte « diese Sprache verstehen (207). ${ }^{12}$ Die Umgangs- und Kommandosprache in dieser Strafkolonie wäre also nicht Französisch - doch was dann? Im Horizont der Zeit Kafkas gab es nur zwei Staaten, die Strafkolonien unterhielten, Frankreich und Russland, doch Russland nur solche in Sibirien. Was außer einer französischen Strafkolonie kam für eine Tropeninsel also infrage? Denn dass die Geschichte in der damaligen Gegenwart spielt, ist offensichtlich - die Hinrichtungsmaschine wird mit zwei elektrischen Batterien betrieben und der Verurteilte erhält, um die Tortur möglichst lange durchzuhalten, Reisbrei aus einem »elektrisch geheizten Napf« (209 u. 219).

Dass der Offizier den Verurteilten »in dessen Sprache « anreden muss (ob auch den Soldaten, bleibt unerklärt), könnte eine Nebensache sein, zöge es nicht die Frage nach sich, ob dieser den Urteilsspruch, der ihm von der Foltermaschine auf den Leib gestochen - geschrieben - werden soll, dann überhaupt verstehen kann. Annehmen lässt sich natürlich, dass auch die Maschine die fremde Sprache benutzt, was wiederum hieße, dass ebenso der Reisende sie beherrschte. Denn nicht nur werden ihm von dem Offizier die Schablonen vorgehalten, auf denen die Urteilssprüche stehen (dass er sie nicht lesen kann, hat nur mit ihrer ornamentalen Gestaltung zu tun), er spricht den Soldaten wie den Verurteilten auch an und wird von ihnen verstanden. Außerdem ist ihm ebenso wie den Einheimischen die Inschrift auf dem Grabstein verständlich, den er sich vor seiner Abreise noch ansieht.

Von einer solchen fremdsprachlichen Welt wird nun allerdings in keiner der Interpretationen ausgegangen, und sie hat im Horizont der zeitgenössischen Leser auch keinen Sinn. Wohl aber wird geschlossen, dass der Verurteilte den für ihn vorgesehenen Schuldspruch, weil auf Französisch, tatsächlich nicht verstehen kann. Er werde ihm »mit einer Sprache auf den Leib geschrieben, die nicht die seine ist und deren Worte, selbst wenn er sie lesen könnte, für ihn gar nicht zu verstehen wären«,

12 Seitenangaben nach Franz Kafka, Drucke zu Lebzeiten, hrsg. Wolf Kittler, Hans-Gerd Koch, Gerhard Neumann, Frankfurt a.M. 2002, 203-248. 
heißt es bei Honold. ${ }^{13}$ Was jedoch müsste man dann folgern? Die Erklärung des Offiziers, es wäre nutzlos, dem Verurteilten das Urteil vorher zu verkünden, denn er erführe es ja »auf seinem Leib« (211), wäre weiter nichts als eine Ausrede zu dem Zweck, den Reisenden über das absolut Unsinnige des Verfahrens zu täuschen. Ebenso wären aber auch die sämtlichen Ausführungen des Offiziers zur Arbeitsweise der Maschine, seine Erklärung ihrer komplizierten Mechanik zur Anlage einer bestimmten Schrift, sein feierliches Beschwören der schließlich erlösenden Erkenntnis usw. lediglich Täuschungen oder Selbsttäuschungen, sofern jedenfalls, wie jederzeit möglich, die Verurteilten kein Französisch könnten.

Eine zutreffende Auslegung? Natürlich nicht, sondern nur ein Beleg dafür, wohin es führt, wenn gegen alle Vernunft an einer Textaussage festgehalten wird, die ersichtlich unverwendbar und mithin ein Fehler ist. Doch würde man sie auch einen »unverwischbaren Fehler« nennen? Das mitnichten. Die Fremdsprachigkeit wird nämlich nur zweimal berührt, ansonsten kommt sie über den ganzen Text hinweg nicht vor. Es müssten also nur an zwei Stellen einige wenige Wörter gestrichen werden, und das Sprachenproblem gäbe es nicht. ${ }^{14}$ Anscheinend hat Kafka das Irritierende dieser Zutat nicht bemerkt, weil es ihm für seinen Verurteilten auf die Fremdsprachigkeit auch nicht ankam. ${ }^{15}$ Wichtig war ihm nur, dass der Mann ungebildet, ahnungslos und kulturfremd ist, damit ihn die technische Raffinesse des Hinrichtungsvorganges umso mehr zu einem bedauernswerten Opfer macht. Wenn sich angesichts seiner Zurückgebliebenheit bezweifeln lässt, dass er überhaupt lesen kann, ist das allerdings des Primitiven zu viel.

Veranlasst zu dem Gegenüber von technischer Modernität und endogener Rückständigkeit wurde Kafka wahrscheinlich durch einen zeitgenössischen Bericht über das französische Strafkoloniesystem im Pazifik, Robert Heindls Buch Meine Reise

\footnotetext{
13 Alexander Honold (Anm. 9), 492 und 495. Genau genommen wird damit nicht nur eine andere Sprache, sondern auch eine andere Schrift (als die lateinische) für diese Sprache vorausgesetzt. Ebenso geht von der Unerschließbarkeit des Urteilstextes schon Walter H. Sokel aus (Franz Kafka, Tragik und Ironie, München 1964, 110). Er nimmt an, dass die Maschine französisch schreibt und die Verurteilten (wohl alle) ihre Botschaften deshalb nicht verstehen. Denselben Schluss zieht Peter Horn, »>Ein eigentümlicher Apparat< im Blick eines Forschungsreisenden«, Acta Germanica 19 (1988), 49-78.

14 Geändert werden müsste die Stelle, dass der Reisende sich über die Gleichgültigkeit des Soldaten wie des Verurteilten bei den Ausführungen des Offiziers nicht wundert, »denn der Offizier sprach französisch und französisch verstand gewiß weder der Soldat noch der Verurteilte.« Das Desinteresse des Soldaten könnte gut oder sogar besser damit begründet werden, dass er die Erklärungen des Offiziers schon viele Male gehört hat. Die Begriffsstutzigkeit des Verurteilten hingegen bedürfte keiner Erklärung, da er ohnehin die Situation nicht versteht. An der zweiten Stelle, dem Satz: »>Du bist frei<, sagte der Offizier zum Verurteilten in dessen Sprache« (237), brauchte nur der Zusatz zur Sprache wegzufallen.

15 Hätte Kafka auf die Sprachdifferenz Wert gelegt, sollte eine Frage des Reisenden nach der Gebrauchssprache der Maschine nicht unterblieben sein, gut anzubringen, als ihm der Offizier die Schablonen zeigt, die er nicht lesen kann. Jede nähere Beleuchtung dieses Punktes könnte aber auf nachgerade parodistische Überformungen hinauslaufen: Kann der Verurteilte überhaupt lesen? Hat seine Sprache eine Schrift? So wichtig, wie die Schrifterkenntnis für den Hinrichtungsvorgang ist, müssten die Verurteilten womöglich erst einen Sprachkurs absolvieren, bevor sie zu ihrer Tötung antreten könnten. Selbstverständlich ist Kafka für die Hinrichtungsprozedur aber von einer schriftgelehrten Sprachgemeinschaft ausgegangen, auch zumal, was das Zuschauerinteresse angeht. Ob eine Strafkolonie und noch dazu ein Eingeborener als Verurteilter diese Voraussetzung erfüllen, hat er sich mit Sicherheit nicht gefragt.
} 
nach den Strafkolonien. ${ }^{16}$ Heindl schildert darin die Verhältnisse in den Strafkolonien von Neukaledonien, einer Inselgruppe im Stillen Ozean östlich von Australien. Das Besondere dieser Inseln, im Unterschied zu den französischen Strafkolonien auf den Inseln vor Südamerika: es gab dort Ureinwohner, Melanesier, von Heindl »Kanaker« genannt, die von den Franzosen zu allen möglichen Hilfsdiensten herangezogen wurden. Ihr Erscheinungsbild und ihre Lebensweise bildete den stärksten Gegensatz zu dem westlich entwickelten Koloniesystem, in dem es auch zu Hinrichtungen mit der Guillotine regelmäßig kam. So bestraft wurden allerdings nicht die Eingeborenen, sondern meistens entlassene Strafgefangene, die auf den Inseln geblieben waren und dort erneut schwere Straftaten begingen. Die Melanesier, die freiwillig in den Dienst der Franzosen traten, erhielten bei Regelverstößen ihre eigene Höchststrafe: Sie wurden entlassen.

Kafkas Verurteilter, »ein stumpfsinniger, breitmäuliger Mensch mit verwahrlostem Haar und Gesicht« (203), entspricht nun ganz und gar den bei Heindl beschriebenen und auch abgebildeten »Kanakern«. Den französischen Wachmannschaften gehörten sie selbstverständlich nicht an, aber man stellte immerhin eine Hilfspolizei aus ihnen zusammen, die entlaufene Sträflinge wieder einfangen sollte. So konnte sich der Eindruck eines gewissen Dienstverhältnisses für sie ergeben, nur dass bei Kafka der Mann doch ein Soldat ist, der »wegen Ungehorsam und Beleidigung des Vorgesetzten verurteilt« wird (203). Allerdings verdunkelt Kafka den Soldatenstand im Verlauf der Geschichte mehr und mehr. Als das Delikt besprochen wird, nämlich dass der Verurteilte den Wachdienst bei einem Hauptmann verschlafen hat, weisen die Begriffe »Diener « und »Herr « schon auf ein eher privates Dienstverhältnis hin (212f.), und dass er in halben Lumpen statt in Uniform zur Hinrichtung geführt wird, lässt ihn auch nicht wie einen Kameraden des ihn begleitenden Soldaten aussehen.

Vollends verwischt wird sein Status aber, wenn der Reisende zu dem Vorgang der Aburteilung überlegt, »daß es sich hier um eine Strafkolonie handelte, daß hier besondere Maßregeln notwendig waren und daß man bis zum letzten militärisch vorgehen mußte.«(214). Doch wie sonst?, wäre zu fragen, da ein Verstoß gegen die militärische Dienstpflicht ja vorliegt. Offenkundig soll dieser Zusammenhang aber nicht mehr wahrgenommen werden. So wird auch kaum jemand noch stutzen, wenn der Offizier, als sich der Verurteilte bei Beginn der Prozedur übergeben muss, dies auf die ihm verabreichten Zuckersachen zurückführt, wo er sich doch »sein ganzes Leben [...] von stinkenden Fischen genährt« hat (223). Die Regelverpflegung der französischen Wachmannschaften kann damit kaum wohl gemeint sein. Vielmehr sieht man den Verurteilten ganz und gar auf den Stand eines unzivilisierten Eingeborenen heruntergebracht, der nicht einmal ein zubereitetes Essen gewohnt ist. Auch dass er den Soldaten nach seiner Freilassung durch einfältige Späße für sich einzunehmen sucht, gehört zu diesem Bild.

\footnotetext{
16 Robert Heindl, Meine Reise nach den Strafkolonien, Berlin, Wien 1913. Hingewiesen hat auf Kafkas wahrscheinliche Kenntnis dieses Buches erstmals Walter Müller-Seidel, Die Deportation des Menschen. Kafkas Erzählung In der Strafkolonie im europäischen Kontext, Stuttgart 1986. Näher ausgeführt findet sich die Verbindung in Klaus Wagenbach (Hrsg.), In der Strafkolonie. Eine Geschichte aus dem Jahr 1914, Berlin 1995.
} 
Nirgendwo deutet die Erzählung jedoch an, dass er ein Sträfling ist. Gerade diese Bezeichnung wird aber immer mehr üblich. ${ }^{17}$ Kafka wusste noch, dass Sträflinge abgeurteilte Straftäter waren, die zum Arbeiten in die Strafkolonien geschickt wurden, sodass sie dort nicht erneut und dann gar zum Tode verurteilt werden konnten. Aufsässigkeiten ebenso wie Fluchtversuche wurden mit Haft oder verschärften Arbeitsbedingungen geahndet, es sollte ja vor allem die Arbeitskraft der Verurteilten genutzt werden. Wenn es bei Kafka heißt, »immer wieder Hafenbauten« stünden für den neuen Kommandanten auf dem Programm (233), deutet sich dieser Hintergrund an. Die Sträflinge selbst jedoch kommen kein einziges Mal vor. Selbst am Schluss, als im Teehaus der Blick auf »armes, gedemütigtes Volk « fällt, handelt es sich nicht um Sträflinge, sondern um »wahrscheinlich Hafenarbeiter « (247). So hat die Schauplatzwahl für Kafka nur den einen Grund: einen Vorstellungsraum für das Hinrichtungsverfahren selbst zu schaffen. Gerüchtweise war bekannt, dass sich in den französischen Strafkolonien Unglaubliches zutrug. Folglich konnte auch die Tötung mit einer solchen Stechmaschine dort in Betracht kommen, wenn sie denn überhaupt irgendwo auf der Welt für möglich zu halten war.

Macht man sich bewusst, zu was für einer Figur der angebliche Soldat im Laufe des Geschehens mutiert, so musste die heikelste Stelle der Erzählung die werden, wo er als Begnadigter in die Kolonie zurückkehrt. Jede Erinnerung an das Hinrichtungsgeschehen in dieser Umgebung hätte einen nicht auflösbaren Widerspruch offengelegt, nämlich den zwischen seiner Ahnungslosigkeit einerseits und der Bekanntheit des Verfahrens in der Kolonie andererseits. Für eine lange Zeit sind die Hinrichtungen gesellschaftliche Großereignisse gewesen. Schon »einen Tag vor der Hinrichtung war das ganze Tal von Menschen überfüllt«, und »vor hunderten Augen [...] wurde der Verurteilte vom Kommandanten selbst unter die Egge gelegt.« (225). Unter dem neuen Kommandanten wird das Spektakel zwar nicht mehr besucht, doch es findet noch regelmäßig statt. ${ }^{18}$ Da man die Verurteilten bedauert,

\footnotetext{
17 Eingeführt hat diese Beurteilung Walter Müller-Seidel. Obwohl er einräumt, dass man in Kafkas Erzählung von den Sträflingen »so gut wie nichts« zu sehen bekomme, scheint ihm die Situation bestimmt von »einem eklatanten Mißverhältnis zwischen Sträflingen und Strafvollzugspersonal« ([Anm. 16], 111). Sicherlich, wenn es keinen Sträfling, jedoch drei Wachsoldaten gibt, trifft das zu. Immerhin könnten sich nach Müller-Seidels Vermutung der Verurteilte und der Soldat aber »vom erniedrigenden Stand des deportierten Häftlings in eine solche Stellung `hinaufgearbeitet haben «, nur werde es »nicht gesagt« ([Anm. 16], 110). Wie über eine so kuriose Prämisse Kafkas Erzählung mit der Deportierungspraxis der europäischen Kolonialmächte in Verbindung gebracht werden kann (ein >Hinaufarbeiten< von Sträflingen zu Wachsoldaten kam selbstverständlich niemals vor), wird immer erstaunlich bleiben, doch für die Kafka-Literatur hatte es Folgen. Bei Martin Beckmann (»Franz Kafkas Erzählung In der Strafkolonie, ein Deutungsversuch «, Wirkendes Wort 39 [1989], 375-392) ist der bei Kafka Verurteilte definitiv ein Sträfling. Klaus Wagenbach (Anm. 16) bezieht zur Kennzeichnung der Handlungsverhältnisse mehrmals historische Sträflingsbilder ein, zeigt aber nur einmal, gleichsam als Beifang, einen Wächter. Ebenso klassifiziert Alexander Honold (Anm. 9) den Verurteilten als Sträfling, von weiteren, beiläufigen Benennungen dieser Art abgesehen. Auf die Deutungen wirkt sich diese Fehleinschätzung zwar nicht aus, aber sie zeigt doch, welcher Kontextverlust für Kafkas Erzählung inzwischen eingetreten ist.

18 Die Häufigkeit der Hinrichtungen wird von dem Offizier allerdings abenteuerlich übertrieben. Wenn er beklagt, dass er »seit einem Vierteljahr « einen neuen Filz erbitte, weil an dem alten »mehr als hundert Männer im Sterben gesaugt und gebissen haben«, müsste man für diesen Zeitraum auf etwa fünfzig Hinrichtungen schließen. Auch dass ein neuer Riemen zum Festschnallen der Verurteilten »erst nach zehn Tagen « geliefert wird, sodass er nicht wisse, »wie er in der Zwischenzeit ohne Riemen die Maschine betreiben soll«, weist auf mehrere Hinrichtungen pro Woche hin (222f.). Da jede Hinrichtung zwölf Stunden
} 
werden ihnen vor ihrer Abführung Süßigkeiten zugesteckt, man erörtert das Unmenschliche der Prozedur und streitet darum, ob sie nicht abgeschafft werden sollte. Wie aber könnte es dann sein, dass ein Bewohner dieser Kolonie, noch dazu ein Soldat, nicht die geringste Ahnung hat, was die Stechmaschine mit ihm anstellen wird?

Wenn Kafka einen »tieferen Mangel« kurz vor dem Ende der Erzählung erkennt und bemerkt, »es ist da irgendwo ein Wurm, der selbst das Volle der Geschichte hohl macht«, so wird er auf eben diese Unvereinbarkeit gestoßen sein. Sie ließ sich nur dadurch verdecken, dass an dem vormaligen Rangunterschied zwischen dem Verurteilten und dem Soldaten nicht festgehalten wird. Und in der Tat: Als die beiden als Begleiter des Reisenden in die Kolonie zurückkommen, fällt kein Wort mehr darüber, dass sich der Verurteilte mit seinen hinten durchgeschnittenen Lumpen vor dem Soldaten eben noch selbst zum Gespött gemacht hat. Beide gehören jetzt dem gleichen Stand an und wissen über die Verhältnisse der Kolonie gleichermaßen Bescheid. Auf die Frage des Reisenden nach dem Grab des alten Kommandanten »liefen beide, der Soldat wie der Verurteilte, vor ihm her und zeigten mit ausgestreckten Händen dorthin, wo sich das Grab befinden sollte.« (247). Über das erwartbare Erstaunen der Anwesenden, dass der Verurteilte wohlbehalten vom Richtplatz zurückkommt, wird notwendigerweise hinweggegangen. Und zu dem nicht vorstellbaren Austausch über das dort Beobachtete heißt es nur noch: »Der Soldat und der Verurteilte hatten im Teehaus Bekannte gefunden, die sie zurückhielten. Sie mußten sich aber bald von ihnen losgerissen haben « (248). Alle Aufmerksamkeit in dieser Situation richtet sich auf den Reisenden und die Frage, wie er die Grabinschrift, die die Wiederkehr des Kommandanten ankündigt, aufnehmen wird. So wird durch die Wahrnehmungslenkung Kafkas das Problem an dieser Stelle nahezu unsichtbar, bleibt aber doch als »unverwischbarer Fehler« bestehen.

Denn der Widerspruch zwischen der notwendigen Ahnungslosigkeit der Verurteilten einerseits und dem Sensationscharakter der Hinrichtungen andererseits durchzieht die gesamte Erzählung. Die Bestrafungen laufen so ab und müssen so ablaufen, dass ein ertappter Übeltäter - »Die Schuld ist immer zweifellos«, erklärt der Offizier (212) - ohne jede Verhandlung in Ketten gelegt und der Hinrichtung zugeführt wird. Erst unter der Stechmaschine erfährt er, weshalb er getötet wird, also welches Gebot er übertreten hat. Er ist deshalb aber äußerst begierig, den Grund zu erfahren, und erlebt die Entzifferung des Schuldspruches als ein höchstes Glück. Der Offizier gerät in der Schilderung dieses Momentes geradezu ins Schwärmen: »Um die Augen beginnt es. Von hier aus verbreitet es sich. Ein Anblick, der einen verführen könnte, sich mit unter die Egge zu legen. Es geschieht ja nichts weiter, der Mann fängt bloß

dauert, also nur eine pro Tag stattfinden kann, und zusätzlich mindestens ein Vorbereitungstag anzunehmen ist, an dem die Maschine gereinigt werden muss, wird die logische Grenze von 180 Hinrichtungen pro Jahr sogar schon überschritten. Nur: wie könnte es bei dieser Anzahl ein beständiges Zuschauerinteresse gegeben haben? Die Einwohner der Kolonie wären, solange sie Anteil genommen haben, zu weiter nichts gekommen, als sich andauernd zwölfstündige Hinrichtungen anzusehen. Und wie wahrscheinlich ist es, dass in einer Strafkolonie jeden zweiten Tag ein Wachsoldat hingerichtet wird? Ein solches System müsste beständig neue Soldaten heranschaffen und könnte doch nicht verbergen, dass deren Lebenserwartung bei Weitem geringer ist als die der Sträflinge. So phantastisch die Angaben des Offiziers aber sind, der Reisende nimmt keinerlei Anstoß an ihnen, sondern überlegt nur, ob er berechtigt sei, »in fremde Verhältnisse entscheidend einzugreifen« (222). 
an, die Schrift zu entziffern, er spitzt den Mund, als horche er.« (219). Gebannt wie er selbst waren aber auch die zahlreichen Zuschauer, die in den guten Zeiten dem Vorgang beiwohnten, »alle wussten: Jetzt geschieht Gerechtigkeit.« Sie kannten offenbar jeweils das Delikt wie den Schuldspruch, mussten beides kennen, und weideten sich an dem »Ausdruck der Verklärung « in dem Gesicht des Übeltäters, wenn ihm selbst der Grund seiner Tötung aufging (226).

Natürlich versteht jeder, dass das nicht für bare Münze zu nehmen ist, sondern »etwas heißen « soll. Trotzdem kann man sich die Frage stellen, woher in einer solchen Kolonie immer wieder neu die Delinquenten kommen sollen, die noch nie von diesen Hinrichtungen gehört haben. Denn wer jemals eine solche zwölfstündige Prozedur gesehen oder von ihr erfahren hat, der wüsste, was ihn erwartet, wenn man ihn vor diese Maschine führt. Und selbst der Schuldspruch! Kann man wirklich glauben, dass Kafkas Verurteilter, wäre er mit diesen Hinrichtungen einmal konfrontiert gewesen, nicht schon ahnen würde, dass man ihm etwas zur Gehorsamspflicht auf den Rücken stechen wird? Es gibt an dieser Stelle einfach keine Botschaft, die eine solche Spannung erzeugen könnte, dass sie dem Hingerichteten mehr bedeuten könnte als sein Leben. Auch sonst ja sind gerichtliche Begründungen für die Verurteilten gemeinhin weniger wichtig als die über sie verhängte Strafe.

Im Horizont der Erzählung geblieben, hätte es nur einen Weg gegeben, das Erstaunen, die Ratlosigkeit, das Aufklärungsbedürfnis der Verurteilten halbwegs glaubhaft einzurichten, und das wäre die vollständige Geheimhaltung der Hinrichtungen gewesen. Das hätte dann auch den einsam getroffenen Urteilen durch den Offizier entsprochen, so wie ja alle Systeme, die ihre Urteile nicht öffentlich verhängen, Geheimdienste, Femegerichte, Sondertribunale, sie auch ohne Zeugen vollstrecken lassen. In dem Roman Der Process, an dem Kafka während der Abfassung der Strafkolonie ebenfalls gearbeitet hat, wird der verdeckt angeklagte K. zum Schluss bei Nacht in einem Steinbruch von zwei Männern abgestochen »wie ein Hund «. Allerdings gäbe es bei geheimen Hinrichtungen die Verankerung im allgemeinen Gerechtigkeitsbedürfnis nicht, und auch die Erklärung der Prozedur gegenüber einem Reisenden müsste entfallen, da das Bekanntwerden der Tötungen gerade nicht erwünscht wäre. Letztlich würde nicht einmal zu verstehen sein, warum überhaupt so aufwändig hingerichtet wird, wenn doch außer dem Durchführenden nie jemand zusieht. Auch hier käme es also zu Widersprüchen, die das Bestrafungsritual insgesamt infrage stellen würden.

Dass Kafka für seine Konstruktion diese logische Unstimmigkeit aber in Kauf nahm, zeigt an, worum es ihm eigentlich ging: Er wollte einen Qualprozess »veröffentlichen«, der anscheinend kein öffentlicher war und der deshalb in einen öffentlichen transformiert werden musste. Welcher das war, ist nicht schwer zu erschließen, und die Kafka-Literatur hat ihn auch längst einvernehmlich bestimmt. Es sind Kafkas eigene Schreibqualen gewesen, die hier verhandelt werden, oft genug von ihm selbst wortwörtlich als Folter bezeichnet. »Ja, das Foltern ist mir äußerst wichtig «, schreibt er 1920 an Milena Jesenská, »ich beschäftige mich mit nichts anderem als Gefoltert-werden und Foltern. Warum? [...] nämlich um aus dem verdammten Mund 
das verdammte Wort zu erfahren. $\ll^{19}$ Da Kafka selbst es aber war, der sich diese Folter auferlegte, kann über die Rollenverteilung in der Erzählung kein Zweifel bestehen. Der Offizier mit seiner weltvergessenen Fixierung auf die Arbeit seiner Maschine, die man auch als »Schreibmaschine« verstehen kann, steht für Kafkas rücksichtsloses, ja borniertes Verfolgen seiner literarischen Mission. ${ }^{20}$ Der Verurteilte aber, der er ebenso ist, muss unter schrecklichen Qualen herausfinden, was diese Mission »meint «, welcher Text erkannt, erschlossen, ins Bewusstsein gehoben werden soll. Die Stunde, in der dem Gepeinigten das aufgeht, bedeutet ihm jedes Mal die größte Erfüllung, selbst der Tod danach hat dann keine Bedeutung für ihn. ${ }^{21}$ Der Reisende aber, der das alles halb ratlos, halb angewidert wahrnimmt, ist der Beobachter in Kafka, der wegen der Ahnung von etwas Großem aber doch zögert, Einspruch zu erheben.

So erschlossen, ist auch klar, warum es Kafka so sehr auf das Nichtwissen des zu erkennenden Textes durch den Verurteilten ankommt und ankommen musste. In der dichterischen Textfindung gibt es für ihn nichts Vorbestimmtes, Vorhersehbares, niemals lässt sich auch nur ahnen, worauf das »Urteil« hinausläuft. Eben in diesem Punkt musste aber auch jede Übertragung in ein öffentliches Geschehen an logische Grenzen stoßen. Es gibt keinen gesellschaftlichen Vorgang, der mit einem solchen individuellen Erkenntnisakt gleichsetzbar ist, auch oder schon gar nicht kommt eine Hinrichtung mit schließlich erfahrener Urteilsbegründung dafür infrage. Dass in den Erläuterungen der Strafkolonie die Nacherzählung des Inhaltes in vielen Fällen breiten Raum einnimmt, oft sogar in rhetorischer Verstärkung, ist ein Indiz. Zusammengefasst erscheinen die Vorgänge unplausibel, es zeigen sich einfach zu viele logische Lücken. Man täte Kafka aber Unrecht, wenn man unterstellte, dass das in allen seinen Werken der Fall ist. Für die Verwandlung, das Urteil oder den Hungerkünstler, aber auch für große Teile seiner Romane lässt sich sehr wohl ein Geschehen skizzieren, das bei Annahme einiger Grundvoraussetzungen in sich selbst stimmig ist. Für die Strafkolonie gilt das jedoch nicht.

Ein Teil dieser Erzählung ist allerdings doch stimmiger und in sich sinnvoller konstruiert, als es in den Interpretationen gemeinhin erkannt wird: die Selbsthinrichtung des Offiziers. Nachdem der Reisende es abgelehnt hat, die Exekutionen mit dem Stechapparat vor den Verwaltungsbeamten der Kolonie zu verteidigen, sieht der Offizier das Ende seiner Hinrichtungsmethode gekommen. Er lässt den Verurteilten frei und will sich selbst mit seiner Maschine töten. Der Urteilsspruch, den er sich einstechen lassen will, passt zu seiner Selbstgewissheit allerdings überhaupt nicht. »Sei gerecht!«, lautet er (238). Da die Maschine dem Verurteilten immer das Gebot

\footnotetext{
19 Franz Kafka, Briefe an Milena, hrsg. Jürgen Born, Michael Müller, Frankfurt a.M. 2004, 290 (Mitte November 1920). Kafkas Folterphantasien behandelt ausführlich Saul Friedlaender, Franz Kafka, München 2012, 136-142.

20 Am deutlichsten wird das herausgestellt bei Detlev Kremer (Anm. 8). Die Verbindung zu Kafkas eigenen Schreibqualen kommt aber in nahezu allen jüngeren Deutungen vor, in der Regel ergänzend zu den ansonsten religiösen, existenzialistischen, zeitkritischen oder psychoanalytischen Zugriffen. Um kein Missverständnis aufkommen zu lassen, sei aber darauf hingewiesen, dass Kafka eine Schreibmaschine nur im Büro benutzt, alle seine Werke aber mit der Hand geschrieben hat.

21 Als eine Spiegelung des Schöpfungsaktes, in dem 1912 Das Urteil entstanden ist, hat die Erzählung gedeutet Mark M. Anderson, Kafka's Clothes, Oxford 1994.
} 
einsticht, das er übertreten hat, bedeutete das, der Offizier wollte sich selbst eines unrechten Tuns beschuldigen. Dafür gibt es jedoch nicht den geringsten Anhaltspunkt. Noch im Tod ist sein Blick »ruhig und überzeugt«, und der Grabstein des vorigen Kommandanten und Erfinders der Maschine kündigt gar die Wiederherstellung des momentan infrage gestellten Strafregimentes an.

Das aber kann nur heißen, das Urteil »Sei gerecht!« ist ein Unrechtsurteil, und es gibt in dem Text selbst dafür auch ein sinnfälliges Indiz. Es ist das Zerbrechen des Degens, nachdem der Offizier sich ausgezogen und alle seine Uniformteile in eine Grube geworfen hat. In das öffentliche Gedächtnis der Kafka-Zeit hatte sich ein solches Ereignis unverwechselbar eingebrannt: die Degradierung des Hauptmanns Dreyfus im Jahr 1895, nachdem man ihn des Verrats von Militärgeheimnissen an die Deutschen bezichtigt hatte. ${ }^{22}$ Der Schuldspruch wurde schon bald angezweifelt, immer mehr erkannt, dass er auf einem antisemitischen Komplott des französischen Offizierskorps beruhte, aber erst 1906 wurde Dreyfus rehabilitiert und in die französische Armee wieder aufgenommen. Die ganze Zeit über aber stand die Szene, da man ihn im Hof der École militaire in Paris degradiert und vor seinen Augen seinen Säbel zerbrochen hatte, der Öffentlichkeit als ein himmelschreiendes Unrecht vor Augen. Bilder davon gingen hunderte Male um die Welt, seine Verbannung in eine Strafkolonie von Guayana war jedermann bekannt, für Kafkas Zeitgenossen konnte der zerbrochene Degen nur bedeuten, dass der Schuldspruch, mit dem der Offizier sich richtet, ein Unrechtsurteil ist.

Vollends beweist sich das dann durch die Art und Weise, wie die Maschine das Urteil an ihm vollstreckt. Sie weigert sich gewissermaßen und zerstört sich im Vorgang seiner Hinrichtung selbst. Das nun allerdings nicht aufgrund eines mysteriösen Eigenwillens, wie von der Fachliteratur ausnahmslos angenommen wird, ${ }^{23}$ sondern weil der Offizier es so einrichtet. Der Text erklärt das eigentlich unübersehbar. Nach dem Einlegen der Schablone mit dem Urteilsspruch ist die Maschine noch keineswegs arbeitsbereit, sondern es müssen umfangreiche Umstellungen an ihr vorgenommen werden. Der Offizier »ordnete das Räderwerk scheinbar gänzlich um; es war eine sehr mühselige Arbeit, es musste sich auch um ganz kleine Räder handeln, manchmal verschwand der Kopf des Offiziers völlig im Zeichner, so genau musste er das Räderwerk untersuchen.«(238). Die Arbeit nimmt so viel Zeit in

\footnotetext{
22 Die Verbindung zum Dreyfus-Fall hat ins Zentrum seiner Interpretation gestellt Sander Gilman, Franz Kafka. The Jewish Patient, New York, London 1995.

23 Die Argumentation zum Versagen der Maschine läuft regelmäßig darauf hinaus, dass der Spruch »Sei gerecht!« von ihr grundsätzlich nicht mitgeteilt werden könne. Die Maschine versagt, schreibt Sokel, »wenn >Gerechtigkeit< als Gebot sich im Zeichner befindet. Der Straf- und Verklärungsapparat ist nicht auf Gerechtigkeit eingestellt« ([Anm. 13], 126). Das ist jedoch gleich zweifach unlogisch. Zum einen verwendet der Offizier eine Originalzeichnung des alten Kommandanten, der die Maschine nicht so präpariert haben wird, dass sie bei einer bestimmten seiner Vorlagen zu Bruch geht. Zum anderen soll ja nicht die Maschine »gerecht « sein, sondern ein Verurteilter darauf hingewiesen werden, dass er gegen diesen Grundsatz verstoßen hat. Gleichwohl wird immer wieder über die Unvereinbarkeit von Maschine und Gerechtigkeit philosophiert und gefolgert, dass überhaupt nichts anderes eintreten könne, als dass der Apparat beim Schreiben dieses Urteils auseinanderfällt. Weitere Äußerungen dieser Art bei Wilhelm Emrich (Franz, Kafka, Bonn 1958, 223), Klaus Mladek (»)Ein eigentümlicher Apparat<. Franz Kafkas In der Strafkolonie«, in: Franz Kafka. Sonderband Text + Kritik, München 1994, 115-142, hier: 134), Oliver Jahraus (Kafka. Leben, Schreiben, Machtapparate, Stuttgart 2006, 337) und vielen anderen.
} 
Anspruch, dass der Verurteilte währenddessen seine verschmutzten Kleider auswaschen kann und dem Reisenden vom langen Hinsehen der Hals steif wird. Dann aber, als »der Offizier oben endlich fertiggeworden war, überblickte er noch einmal lächelnd das Ganze in allen seinen Teilen, schlug diesmal den Deckel des Zeichners $\mathrm{zu}$, der bisher offen gewesen war « und bereitet sich selbst mit dem abschließenden Zerbrechen seines Degens auf die Hinrichtung vor (239).

Deutet so nicht alles darauf hin, dass er weiß, was mit der Maschine geschehen wird? Wie vollkommen er sie beherrscht, wird auch bei seinen weiteren Vorbereitungen noch einmal erklärt: »Wenn schon früher deutlich gewesen war, daß er die Maschine gut verstand, so konnte es jetzt einen fast bestürzt machen, wie er mit ihr umging und wie sie gehorchte.«(241). Sie arbeitet nun auch völlig geräuschlos, bis plötzlich etwas Unerwartetes geschieht: »Langsam hob sich der Deckel des Zeichners und klappte dann vollständig auf. Die Zacken eines Zahnrades zeigten und hoben sich, bald erschien das ganze Rad, [...] drehte sich bis zum Rand des Zeichners, fiel hinunter, kollerte aufrecht ein Stück im Sand und blieb dann liegen.« (243f.). Es ist geradezu eine Vorstellung, mit der die Maschine ihre Zerlegung in Szene setzt, und es kann nichts anderes gemeint sein, als dass es eben die lange Umstellung durch den Offizier ist, die das bewirkt. Er verwendet ja doch eine Zeichnung des alten Kommandanten, die automatisch von der Maschine soll gelesen werden können. Warum also lächelt er, als er seine Arbeit noch einmal überblickt, warum schließt er den Deckel, »der bisher offen gewesen war «? Die Nichtausführung des Urteilsspruches, der »kurze Prozess « und die Selbstzerstörung der Maschine - alles ist sein Werk, weil er die Abschaffung seines Straf- und Belehrungssystems nicht erleben will.

Erstaunlich ist allerdings, dass dieser Zusammenhang anscheinend noch nie bisher bemerkt worden ist. Der Grund dafür ist aber erschließbar: Auch der Reisende als Zeuge bemerkt ihn nicht. Als die Maschine ihre Arbeitsweise ändert und den Offizier zu zerstechen beginnt, will er sie zum Stehen bringen: »das war ja keine Folter, wie sie der Offizier erreichen wollte, das war unmittelbarer Mord.« Und damit nicht genug, hofft er sogar noch, ein »Zeichen der versprochenen Erlösung « im Gesicht der Leiche zu entdecken. Doch es ist, »wie es im Leben gewesen war, [...] was alle anderen in der Maschine gefunden hatten, der Offizier fand es nicht.« (244f.). Schon früh hat Sokel darauf hingewiesen, dass die Erwartung einer Erlösung schon deshalb irrig ist, weil der Offizier den Spruch kennt, den alle anderen Verurteilten erst von der Maschine erfahren. ${ }^{24}$ Doch das ist nur eine Kleinigkeit gegenüber dem Sachverhalt, dass die ganze Prozedur bei dem Offizier kaum länger als eine Viertelstunde dauert, während doch die Entzifferung der Schrift erst nach sechs Stunden möglich werden soll. Wie lässt sich verstehen, dass diese mehrmals mitgeteilte Bedingung von dem Reisenden nicht mehr gewusst wird, wenn schon er nicht durchschaut, dass sich der Offizier das »Sei gerecht! « gar nicht einstechen lassen wollte?

Ein Grund dafür findet sich nicht, ist doch der Reisende stets als ein überlegener Beurteiler der geschilderten Vorgänge gekennzeichnet. Fehleinschätzungen durch ihn werden nicht angedeutet, seine Sicht und die des Erzählers gehen immer wieder

\footnotetext{
24 Sokel (Anm. 13), 129.
} 
ineinander über ${ }^{25}$ Für den Offizier gilt das im Gegensatz zu ihm nicht. Er wirkt so besessen von seiner Hinrichtungsmethode, dass gegenüber allem, was er äußert, Vorsicht angebracht erscheint. Bei offenkundigen Unwahrheiten aus seinem Mund kann man allerdings auch an dieser Einschätzung wieder zweifeln. Wenn er erklärt, er habe angeordnet, dass an die Verurteilten »einen Tag vor der Exekution kein Essen mehr verabfolgt « werden dürfe, sehe aber, dass auch diesmal wieder dagegen verstoßen worden sei (223), so sollte es an irgendeinem Fragezeichen nicht fehlen. Schließlich hat er den Mann nach eigenem Bekenntnis gerade erst am Morgen schuldig gesprochen und ihn sofort der Hinrichtung zugeführt (212). Ein solches Fragezeichen jedoch gibt es nicht, der Reisende bemerkt zu dem Widerspruch nichts. Irrt sich der Offizier hier also, lügt er gar und sollen wir erkennen, dass der Reisende nicht bei der Sache ist? ${ }^{26}$ Oder irrt sich der Autor Kafka?

Wohl eher das Zweite, da für den Reisenden keinerlei Schwäche angezeigt wird. »In den Augen des Reisenden - und daher in unseren«, leitet Sokel eine Reihe von Folgerungen ein, die aus der Strafkolonie-Erzählung seiner Meinung nach zu ziehen sind. ${ }^{27}$ Genauer betrachtet äußert sich allerdings auch der Reisende nicht immer plausibel. Wie für die meisten Figuren Kafkas ergeben sich auch über ihn, also seine Perspektive, mehrmals jene kaum merklichen Verschiebungen in den Sachverhalten, die für Kafkas Erzählweise so kennzeichnend sind. Schon dass er, wie bereits berührt, die Hinrichtung des Verurteilten halb und halb damit entschuldigt, dass man in einer Strafkolonie »bis zum letzten militärisch vorgehen mußte«, obwohl ein anderes Vorgehen gegenüber einem Soldaten überhaupt nicht infrage kommt, gehört hierher. »Immerhin mußte er sich sagen«, wird seine Überlegung eingeleitet (214). Musste sich dieser »Forscher des Abendlandes« das jedoch wirklich sagen? Müsste er sich nicht vielmehr sagen, dass ein Todesurteil bloß für eine Disziplinlosigkeit auch in einer Strafkolonie zu weit geht? Offenbar soll das Urteil selbst jedoch keine Sache des Bedenkens für ihn sein. Alle seine Überlegungen haben nur mit dem formalen Vorgehen des Offiziers zu tun.

Das wird noch deutlicher, wenn er, nachdem ihm die Hinrichtungsmaschine bereits in allen Einzelheiten erklärt worden ist, den Offizier fragt: »Wie lautet denn

\footnotetext{
25 Jede Untersuchung der Erzählperspektive ergibt zwar, dass der Standort des Erzählers nicht genau auszumachen ist, für insgesamt ungewiss wird das Geschehen deshalb aber nicht gehalten. Für Ingeborg Henel erweisen sich sogar »beide Hauptpersonen [...] als genaue und zuverlässige Beobachter«. Widersprüche in den Äußerungen des Offiziers bemerkt sie nicht, und zu all dem Unwahrscheinlichen, das er äußert, heißt es: »Wenn die kühnste Behauptung des Offiziers von einem, der gewissermaßen sein Gegner ist, für wahr gehalten wird, darf auch der Leser dem Offizier glauben und alles, was er sagt, als objektive Wahrheit verstehen.« (»Kafkas In der Strafkolonie«, in: Untersuchungen zur Literatur als Geschichte. Festschrift für Benno von Wiese, hrsg. Vincent J. Günther u. a., Berlin 1973, 480-504, hier: 484).

${ }^{26}$ Die Unstimmigkeit in der Äußerung des Offiziers, dass er angeordnet habe, den Verurteilten einen Tag vor der Hinrichtung nichts mehr zu essen zu geben, obwohl es im aktuellen Fall einen solchen Zeitabstand gar nicht gibt, ist von verschiedenen Interpreten bemerkt worden. Abgeleitet wird daraus jedoch nur der »Eindruck einer mangelhaften Glaubwürdigkeit« für den Offizier, nicht auch für den Reisenden, obwohl diesem der Widerspruch ja doch hätte auffallen sollen. »Um so heller leuchtet das Bild des Reisenden, das anscheinend nicht durch solche Glaubwürdigkeitsmängel beeinträchtigt ist«, urteilt Axel Hecker (An den Rändern des Lesbaren. Dekonstruktivistische Lektüren zu Franz Kafka, Wien 1994, 101). Hier wie auch sonst wird der Reisende als verlässlicher Beurteiler aller Vorgänge angesehen, eine Hinterfragung seiner Wahrnehmungen kommt für keinen der Interpreten in Betracht.
}

27 Sokel (Anm. 13), 125. 
das Urteil? « Dass es sich um die »Exekution eines Soldaten « handelt, der einen Vorgesetzten angegriffen hat, ist ihm mitgeteilt worden - was kann er mit seiner Frage dann noch meinen? Zwar wundert sich darüber auch der Offizier - »Sie wissen auch das nicht? « - , ignoriert die Frage aber und holt aus zu einer Belehrung über die »Form unseres Urteils« und die »Urteilsarten«, d. h. leitet über zu der besonderen Art der Übermittlung des Schuldspruches (209f.). Korrekt hätte er antworten können: Das Urteil lautet »Tod durch Verbluten«, so wie es auch »Tod durch den Strang « oder »Tod durch Erschießen« hätte lauten können. Selbstverständlich sollen wir daraus aber nicht folgern, dass der Reisende, der sich die Umdeutung seiner Frage widerspruchslos gefallen lässt, nicht weiß, worauf es bei der Ausführung eines Todesurteils ankommt. Die Vagheit seiner Frage ist vielmehr ein Mittel des Erzählers Kafka, die Tötung in den Hintergrund treten zu lassen und nur noch die Folter zu behandeln.

Etwas anderes gilt allerdings für den undurchschaut bleibenden Vorgang der Selbsthinrichtung. Es würde an dem Gesamteindruck überhaupt nichts ändern, wenn der Reisende das Geschehen richtig beobachtete und einschätzte. Schon eine einzige Andeutung durch ihn würde genügen - etwa, dass ihm der lange Umbau der Maschine verdächtig vorkommt -, um auch »dem Leser « den wahren Zusammenhang aufzuschließen. Einzuwenden, dass Kafka dann wohl wirklich seine Urteilsfähigkeit zuletzt in Zweifel ziehen will, hätte wenig Sinn. Nicht nur fehlt es auch dafür an einem Hinweis, es entzöge dem Text auch noch jede Verstehensgrundlage. Welche Sachverhalte wären der Fall, welche Täuschung, welche Selbsttäuschung? Für Kafka gilt noch nicht, was in der jüngeren experimentellen Literatur mitunter vorkommt: ein absichtlich auf Verwirrung angelegtes Erzählen. Kein Autor würde schreiben, es sei da »irgendwo ein Wurm, der selbst das Volle der Geschichte hohl macht « ${ }^{28}$, wenn er ein freies, beliebiges Verstehen, also gar kein »Verstehen« beabsichtigt hätte. Auch also das Versagen des Reisenden, das Offensichtliche richtig einzuschätzen, kann als ein Fehler, in diesem Falle erzähltechnischer Art, bezeichnet werden, da es zu der Figur nicht passt und selbst die sorgfältigsten Leser zu falschen Schlüssen veranlasst.

Die vielen verschiedenen, ja gegensätzlichen Deutungen, die von der StrafkolonieErzählung vorliegen, lassen sich durch die Aufdeckung ihrer inneren Unstimmigkeiten allerdings nicht neu sortieren. Ein großer Teil von ihnen beruht gar nicht auf einer genauen Textaufnahme. Oftmals geht es den Interpreten nur darum, ein bereits vorhandenes, aus dem Gesamtwerk abgeleitetes Kafka-Bild auf diese Erzählung zu übertragen. Oder anders formuliert: Es geht ihnen nicht um das Verständnis dieses oder jenes Textes, es geht ihnen um das Verstehen des Autors. Der Nachweis irgendwelcher Widersprüche kann deshalb für oder gegen solche Textausschöpfungen nichts beweisen. Zweifellos erklärt sich diese Art Umgang aus dem Kafka'schen Werk selbst, das größtenteils aus Fragmenten besteht, die eine abschließende Ausgestaltung gar nicht erfahren haben. Die Erzählung In der Strafkolonie allerdings hat sie erfahren, und so sollte man sie als eigenständige Arbeit auch ernst nehmen. Wenn dabei aber sichtbar wird, dass es eine ganze Anzahl von Ungereimtheiten in ihr gibt, unverwischbare wie verwischbare, ist es sinnvoller, sich diese aufzuhellen

28 Kafka (Anm. 7). 
und sie sich zu erklären, als sie mit großartigen Sinnzuschreibungen zuzudecken. Es mag kein Argument sein: aber Kafka wäre mit einem solchen genauen Lesen sehr einverstanden gewesen.

Funding Open Access funding provided by Projekt DEAL.

Open Access Dieser Artikel wird unter der Creative Commons Namensnennung 4.0 International Lizenz veröffentlicht, welche die Nutzung, Vervielfältigung, Bearbeitung, Verbreitung und Wiedergabe in jeglichem Medium und Format erlaubt, sofern Sie den/die ursprünglichen Autor(en) und die Quelle ordnungsgemäß nennen, einen Link zur Creative Commons Lizenz beifügen und angeben, ob Änderungen vorgenommen wurden.

Die in diesem Artikel enthaltenen Bilder und sonstiges Drittmaterial unterliegen ebenfalls der genannten Creative Commons Lizenz, sofern sich aus der Abbildungslegende nichts anderes ergibt. Sofern das betreffende Material nicht unter der genannten Creative Commons Lizenz steht und die betreffende Handlung nicht nach gesetzlichen Vorschriften erlaubt ist, ist für die oben aufgeführten Weiterverwendungen des Materials die Einwilligung des jeweiligen Rechteinhabers einzuholen.

Weitere Details zur Lizenz entnehmen Sie bitte der Lizenzinformation auf http://creativecommons.org/ licenses/by/4.0/deed.de. 